\title{
Magnetic field amplification in newly-born neutron stars
}

\author{
H.-J. Wiebicke and U. Geppert \\ Max-Planck-Institut für extraterrestrische Physik, Außenstelle Berlin, \\ Rudower Chaussee 5, D-12489 Berlin, Germany
}

\begin{abstract}
We present a scenario of magnetic field (MF) evolution of newly-born neutron stars (NSs). Numerical calculations show that in the hot phase of young NSs the MF can be amplified by thermoelectric effects, starting from a moderately strong seed-field. Therefore, there is no need to assume a $10^{12} \mathrm{G}$ dipole field immediately after the gravitational collapse of the supernova (SN) event. The widely accepted scenario for such a field to be produced by flux conservation during the collapse is critically discussed. Instead, it can be generated by amplification and selection effects in the first $10^{4} \mathrm{yrs}$, and by the subsequent fast ohmic decay of higher multipole components, when the NS cools down.
\end{abstract}

\section{Introduction}

The motivation for our approach was the fact, that the origin of the strong MFs of pulsars is still quite unclear. The naive, but widely accepted scenario to generate these fields by flux conservation during the collapse is questionable by several reasons: (a) The MFs of NS progenitors are not well known and probably not of dipole type. (b) The outer shells of the progenitors, which might carry a large portion of their MFs, are blown away in the SN event. (c) The gravitational collapse is certainly not so regular and symmetric as necessary for generating a dipole field.

For the last point there are observational as well as numerical hints: (a) ROSAT observations of the VELA SN remnant show several explosion fragments indicating strong deviations from radial symmetry (Aschenbach et al.). (b) Computer simulations of a $\mathrm{SN}$ explosion show clearly small-scale structures in the explosion cloud and strong irregularities of matter falling back to the proto-neutron star (Janka \& Mïller). Therefore, it is much more natural to assume a complicated mixture of different field modes (toroidal/poloidal; largescale/small-scale) immediately after the collapse, instead of a pure dipole field. But, how to explain then the observations?

As an alternative we propose a scenario, where the MF undergoes an evolution after the NS birth. In this scenario selected MF modes can be amplified by thermoelectric effects up to strengths of $10^{11-12} \mathrm{G}$ in the first $10^{3-4} \mathrm{yrs}$. By a subsequent relatively fast ohmic decay of the higher multipole components the large-scale modes like the dipole and quadrupole ones become dominating. Therefore, there is no need to assume a $10^{12} \mathrm{G}$ dipole field immediately after the gravitational collapse. 


\section{Formalism and basic assumptions}

The thermoelectric effect together with the thermo-Hall effect provides a mechanism for amplification of NS MFs after the NS birth. Both effects are particularly strong in the liquid layer of the NS crust, since in this region (a) the temperature gradient is extremely large $\left(\simeq 10^{5} \mathrm{~K} / \mathrm{cm}\right)$, and (b) the transport coefficients (heat and electric conductivity, thermopower) are highly anisotropic. Therefore, we assume that the pulsar magnetic field is generated mainly in the liquid layer of the outer NS crust. The thermoelectric effect is strong, as long as the NS is hot enough $\left(z 3 \cdot 10^{6} \mathrm{~K}\right)$. Further assumptions are: (a) stationary heat flux through the crust in the first $10^{3}$ yrs, (b) axial symmetry, and (c) hydrodynamic motions can be neglected. Assumption (c) becomes questionable for strong induced MFs $\left(\gtrsim 10^{11} \mathrm{G}\right)$.

As basic equations the heat transport equation and the induction equation were solved simultaneously. They are coupled by the field dependend transport coefficients and the thermoelectric term in the induction equation. Both equations are nonlinear. Toroidal and poloidal field components were introduced, and, together with the temperature variable, expanded in spherical harmonics.

\section{Main results}

Starting from moderately strong seed-fields, small-scale toroidal fields begin to grow exponentially (in a timescale of years), if the following criterion is fulfilled: $T_{\mathrm{s} 6}^{4} / g_{\mathrm{s} 14} \gtrsim 100\left(T_{\mathrm{s} 6}\right.$ - surface temperature in $10^{6} \mathrm{G}, g_{\mathrm{s} 14}$ - surface gravity in $\left.10^{14} \mathrm{~cm} \mathrm{~s}^{-2}\right)$. This corresponds to the linear regime of the differential equations (valid for MF strengths $\lesssim 10^{11} \mathrm{G}$ ). Later they are saturated by nonlinear effects, and maximum MF strengths of some $10^{12} \mathrm{G}$ are reached. Large-scale modes cannot grow in the linear regime, but they are induced by nonlinear interaction with the small-scale ones. The growth rate is even twice of that of the smallscale toroidal fields. Even-n toroidal modes are particularly strongly induced. For example, the quadrupole mode grows until a maximum field strength of some $10^{11} \mathrm{G}$. Poloidal field components are not strongly induced in the present stage of the formalism due to a weak interaction with small-scale toroidal fields. For this aim, hydrodynamic equations should be included in the formalism. For more details see the references below.

\section{References}

Aschenbach, B., Egger, R. \& Trümper, J. 1995, Nature, 373, 587

Geppert, U. \& Wiebicke, H.-J. 1991, A\&AS, 87, 217

Geppert, U. \& Wiebicke, H.-J. 1995, A\&A, 300, 429

Janka, H.-T. \& Müller, E. 1994, A\&A, 290, 496

Wiebicke, H.-J. \& Geppert, U. 1991, A\&A, 245, 331

Wiebicke, H.-J. \& Geppert, U. 1992, A\&A, 262, 125

Wiebicke, H.-J. \& Geppert, U. 1995, A\&A, 294, 303

Wiebicke, H.-J. \& Geppert, U. 1996, A\&A, in press 